TECHNION-PH-2000-28

EFI 2000-34

hep-ph/0010237

October 2000

\title{
U-Spin Symmetry in Doubly Cabibbo-Suppressed Charmed Meson Decaysit
}

\author{
Michael Gronau \\ Department of Physics, Technion-Israel Institute of Technology \\ Technion City, 32000 Haifa, Israel \\ and \\ Jonathan L. Rosner \\ Enrico Fermi Institute and Department of Physics \\ University of Chicago, Chicago, Illinois 60637
}

\begin{abstract}
We prove a U-spin amplitude triangle relation among doubly Cabibbosuppressed (DCS) charmed meson decays, $D^{0} \rightarrow K^{+} \pi^{-}, D^{0} \rightarrow K^{0} \pi^{0}$ and $D_{s}^{+} \rightarrow K^{0} K^{+}$, congruent to an isospin relation among corresponding Cabibbo-favored (CF) decays. U-spin breaking in relative phases between $\mathrm{CF}$ and DCS amplitudes affects time-dependent studies of $D^{0}-\bar{D}^{0}$ mixing. Comparison of final state phase patterns in DCS and CF amplitude triangles, which can shed some light on these phases, is carried out in a phenomenological framework incorporating resonance contributions.
\end{abstract}

Recently the CLEO Collaboration reported a measurement of the DCS decay $D^{0} \rightarrow K^{+} \pi^{-}$[国]. The measured branching ratio, based on a time-dependent rate measurement, is a substantial advance in sensitivity and is considerably lower than the previous world average [2]. The new world average [3] (we use $\tan \theta_{c}=0.2256 \pm 0.0024$ )

$$
\frac{\mathcal{B}\left(D^{0} \rightarrow K^{+} \pi^{-}\right)}{\mathcal{B}\left(D^{0} \rightarrow K^{-} \pi^{+}\right)}=(1.47 \pm 0.31) \tan ^{4} \theta_{c},
$$

is consistent at $90 \%$ confidence level with flavor $\mathrm{SU}(3)$ symmetry, which predicts a value of $\tan ^{4} \theta_{c}$ for the ratio of branching ratios 佃.

Early predictions based on factorization [5], in which several SU(3) breaking effects were claimed to accumulate, were larger than the $\mathrm{SU}(3)$ limit by a factor of about two

\footnotetext{
${ }^{1}$ To be published in Physics Letters B.
} 
to three. An uncertain factor in this estimate is the ratio of form factors $F_{0}^{D K} / F_{0}^{D \pi}$ at low $q^{2}$, which was taken to be smaller than one, whereas a value slightly larger than one is preferred on theoretical grounds. The present average value obtained from four experiments [6] is $F_{0}^{D K}(0) / F_{0}^{D \pi}(0)=1.00 \pm 0.08$. The central value implies a factorization prediction of $1.72 \tan ^{4} \theta_{c}$ which is consistent with (1). However, the use of factorization in $D$ decays has been frequently questioned, most recently in [7], where nearby resonances at 1430 and around 1800-1900 MeV were shown to contribute sizably to $D^{0} \rightarrow K^{-} \pi^{+}$[8].

$\mathrm{SU}(3)$ was observed to be badly broken in several singly Cabibbo-suppressed $\Delta S=0 D$ decays [9]. This includes the ratio of amplitudes [10] $\sqrt{2} \mid A\left(D^{+} \rightarrow\right.$ $\left.\pi^{+} \pi^{0}\right)|/| A\left(D^{+} \rightarrow \bar{K}^{0} \pi^{+}\right) \mid=(1.78 \pm 0.26) \tan \theta_{c}$, which is expected to be $\tan \theta_{c}$ in the $\mathrm{SU}(3)$ limit. Since this ratio consists of decay amplitudes to exotic final states involving $\pi \pi$ in $I=2$ and $K \pi$ in $I=3 / 2$, it demonstrates that $\mathrm{SU}(3)$ breaking is not due only to resonance contributions. An interesting question is whether $\mathrm{SU}(3)$ breaking in DCS $\Delta S=-\Delta C$ processes, which do involve resonances, is in general smaller than in $\Delta S=0$ decays, as seems to be the case in (1). We will study this question in the presence of resonance contributions.

The question of $\mathrm{SU}(3)$ breaking in DCS charmed meson decays plays an important role in time-dependent studies of $D^{0}-\bar{D}^{0}$ mixing. Interference between mixing and decay to "wrong sign" $K \pi$ depends on the strong phase difference $\delta$ between the amplitudes of $D^{0} \rightarrow K^{+} \pi^{-}$and $D^{0} \rightarrow K^{-} \pi^{+}$. A priori knowledge of $\delta$ would simplify the analysis considerably [11]. This phase vanishes in the flavor $\mathrm{SU}(3)$ limit [1, 12]. Theoretical estimates of $\delta$, based on various assumptions about $\mathrm{SU}(3)$ breaking, are strongly model-dependent [13]. Therefore, one seeks model-independent information about this phase, or about any other relative strong phase between corresponding DCS and CF $D^{0}$ decay amplitudes.

In the present Letter we study various tests of $\mathrm{SU}(3)$ symmetry in DCS charmed meson decays. We reconsider a variety of U-spin relations [14] between CF and DCS decay rates, some of which were proposed twenty five years ago [四]. In particular, we prove an amplitude triangle relation among DCS decays, $D^{0} \rightarrow K^{+} \pi^{-}, D^{0} \rightarrow K^{0} \pi^{0}$ and $D_{s}^{+} \rightarrow K^{0} K^{+}$, congruent to an isospin triangle relation among corresponding $\mathrm{CF}$ decays. U-spin breaking would violate this relation, and could modify the finalstate phase pattern of DCS processes relative to that of CF decays. A comparison of these two patterns is shown to indirectly shed light on the magnitude of relative final state phases between CF and DCS amplitudes. We study these patterns within a phenomenological framework which incorporates resonant contributions in an $\mathrm{SU}(3)$ breaking fashion. Using a range of $\mathrm{SU}(3)$ breaking parameters, we show that the phase $\delta$ can be as large as about 20 degrees. We point out certain experimental difficulties in measuring final state phases in DCS decays.

The presence of non-trivial relative phases between the amplitudes contributing to certain CF D meson decays can be ascertained by constructing amplitude triangles based on experimentally observed decay rates. The subprocess $c \rightarrow s u \bar{d}$ involves a $\Delta I=1$ transition. The amplitudes of the three two body decays $D^{0} \rightarrow K^{-} \pi^{+}$, $D^{0} \rightarrow \bar{K}^{0} \pi^{0}$, and $D^{+} \rightarrow \bar{K}^{0} \pi^{+}$are governed by two isospin amplitudes corresponding 
to $I=1 / 2$ and $I=3 / 2$ final states. Thus, the square roots of the corresponding rates form a triangle

$$
A\left(D^{0} \rightarrow K^{-} \pi^{+}\right)+\sqrt{2} A\left(D^{0} \rightarrow \bar{K}^{0} \pi^{0}\right)=A\left(D^{+} \rightarrow \bar{K}^{0} \pi^{+}\right) .
$$

Similar triangle relations hold in quasi two-body decays into a vector and a pseudoscalar meson:

$$
\begin{aligned}
A\left(D^{0} \rightarrow K^{*-} \pi^{+}\right)+\sqrt{2} A\left(D^{0} \rightarrow \bar{K}^{* 0} \pi^{0}\right) & =A\left(D^{+} \rightarrow \bar{K}^{* 0} \pi^{+}\right) \\
A\left(D^{0} \rightarrow \rho^{+} K^{-}\right)+\sqrt{2} A\left(D^{0} \rightarrow \rho^{0} \bar{K}^{0}\right) & =A\left(D^{+} \rightarrow \rho^{+} \bar{K}^{0}\right)
\end{aligned}
$$

and for partial-wave amplitudes $A_{l}$ in decays to two vector mesons:

$$
A_{l}\left(D^{0} \rightarrow K^{*-} \rho^{+}\right)+\sqrt{2} A_{l}\left(D^{0} \rightarrow \bar{K}^{* 0} \rho^{0}\right)=A_{l}\left(D^{+} \rightarrow \bar{K}^{* 0} \rho^{+}\right)
$$

where relations hold separately for S, P and D-waves.

If a triangle has non-zero area, the two corresponding isospin amplitudes have a non-trivial phase with respect to one another. Using experimental data, $I=1 / 2$ and $I=3 / 2$ amplitudes were shown [15, 16] to have relative phases close to $90^{\circ}$ for the decays $D \rightarrow \bar{K} \pi$ and $D \rightarrow \bar{K}^{*} \pi$, but near zero for $D \rightarrow \rho \bar{K}$.

The isospin decomposition in DCS $\Delta S=-\Delta C$ processes differs from that in $\mathrm{CF} \Delta S=\Delta C$ decays. In the former case the quark subprocess $c \rightarrow d u \bar{s}$ involves both $\Delta I=0$ and 1 transitions which yield three isospin amplitudes for $I=1 / 2$ and $I=3 / 2$ final states. There are four allowed charge states in $D^{0}$ and $D^{+}$two body decays: $D^{0} \rightarrow K^{+} \pi^{-}, D^{0} \rightarrow K^{0} \pi^{0}, D^{+} \rightarrow K^{+} \pi^{0}$ and $D^{+} \rightarrow K^{0} \pi^{+}$. The four physical amplitudes, which are linear combinations of the three isospin amplitudes, obey a quadrangle relation

$$
A\left(D^{0} \rightarrow K^{+} \pi^{-}\right)+\sqrt{2} A\left(D^{0} \rightarrow K^{0} \pi^{0}\right)=A\left(D^{+} \rightarrow K^{0} \pi^{+}\right)+\sqrt{2} A\left(D^{+} \rightarrow K^{+} \pi^{0}\right) .
$$

Similar quadrangle relations apply to quasi-two body decays into pairs of a vector and pseudoscalar meson:

$$
\begin{aligned}
A\left(D^{0} \rightarrow K^{*+} \pi^{-}\right)+\sqrt{2} A\left(D^{0} \rightarrow K^{* 0} \pi^{0}\right) & =A\left(D^{+} \rightarrow K^{* 0} \pi^{+}\right)+\sqrt{2} A\left(D^{+} \rightarrow K^{*+} \pi^{0}\right) \\
A\left(D^{0} \rightarrow \rho^{-} K^{+}\right)+\sqrt{2} A\left(D^{0} \rightarrow \rho^{0} K^{0}\right) & =A\left(D^{+} \rightarrow \rho^{+} K^{0}\right)+\sqrt{2} A\left(D^{+} \rightarrow \rho^{0} K^{+}\right),
\end{aligned}
$$

and to partial-wave amplitudes into $K^{*} \rho$ states.

These quadrangle relations are quite different from the isospin triangles in $\mathrm{CF}$ decays. As we will see now, relations between two sides of the CF triangle (2) and two sides of the DCS quadrangle (6) follow from an approximate U-spin symmetry, thereby permiting in this approximation a triangle construction also for DCS decays. Similar relations are obeyed by $\mathrm{S}$ and $\mathrm{D}$ wave amplitudes in decays to $K^{*} \rho$, but do not hold for decays into a vector and a pseudoscalar meson.

A discrete U-spin symmetry transformation, interchanging $d$ and $s$ quarks, implies simple relations between $\Delta S=\Delta C$ and $\Delta S=-\Delta C$ processes [4]. This transformation interchanges the four-quark $U=1$ transition operators, $c \rightarrow s u \bar{d}$ and $c \rightarrow d u \bar{s}$, 
and implies $D^{0} \leftrightarrow D^{0}, D^{+} \leftrightarrow D_{s}^{+}$and $\pi^{ \pm} \leftrightarrow K^{ \pm}, \bar{K}^{0} \leftrightarrow K^{0}$ in initial and final states. The $\bar{K}^{0} \pi^{0}$ final state is a special case. The two pseudoscalars which are in an S-wave are in a symmetric U-spin state. The $\bar{K}^{0}$ is $U=1$, while the $\pi^{0}$ is a combination of $U=0$ and $U=1$. In $D^{0} \rightarrow \bar{K}^{0} \pi^{0}$ the $\Delta U=1$ transition leads to a $U=1$ final state to which only the $U=0$ component of the $\pi^{0}$ contributes. Thus, in $D^{0}$ decay U-spin reflection implies $\bar{K}^{0} \pi^{0} \leftrightarrow K^{0} \pi^{0}$.

A general U-spin prediction is that the ratio of every pair of U-spin related DCS and CF decay amplitudes is given by the CKM factor $V_{c d}^{*} V_{u s} / V_{c s}^{*} V_{u d}=-\tan ^{2} \theta_{c}$. Hence one finds [4]

$$
\begin{aligned}
\frac{A\left(D^{0} \rightarrow K^{+} \pi^{-}\right)}{A\left(D^{0} \rightarrow K^{-} \pi^{+}\right)} & =\frac{A\left(D^{0} \rightarrow K^{0} \pi^{0}\right)}{A\left(D^{0} \rightarrow \bar{K}^{0} \pi^{0}\right)}=\frac{A\left(D^{+} \rightarrow K^{0} \pi^{+}\right)}{A\left(D_{s}^{+} \rightarrow \bar{K}^{0} K^{+}\right)} \\
& =\frac{A\left(D_{s}^{+} \rightarrow K^{0} K^{+}\right)}{A\left(D^{+} \rightarrow \bar{K}^{0} \pi^{+}\right)}=-\tan ^{2} \theta_{c}
\end{aligned}
$$

Note that this not only predicts the ratios of magnitudes for the corresponding amplitudes, but also implies equal final state phases in CF and in DCS decays. In this approximation, the quadrangle relation Eq. (6) breaks into two triangle relations

$$
\begin{aligned}
A\left(D^{0} \rightarrow K^{+} \pi^{-}\right)+\sqrt{2} A\left(D^{0} \rightarrow K^{0} \pi^{0}\right) & =A\left(D_{s}^{+} \rightarrow K^{0} K^{+}\right) \\
A\left(D^{+} \rightarrow K^{0} \pi^{+}\right)+\sqrt{2} A\left(D^{+} \rightarrow K^{+} \pi^{0}\right) & =A\left(D_{s}^{+} \rightarrow K^{0} K^{+}\right)
\end{aligned}
$$

The situation in decays to pairs of a vector and a pseudoscalar meson $(V P)$, $D \rightarrow K^{*} \pi$ and $D \rightarrow \rho K$, is different. Here the two mesons in the final states are in a P-wave and both $U=0$ and $U=1$ components of the $\pi^{0}$ or $\rho^{0}$ contribute. Consequently, ratios similar to Eq. (8) do not apply to $V P$ final states involving these neutral mesons, and the quadrangles (7) do not break into two triangles. Certain ratios of DCS to CF amplitudes are still given in the U-spin symmetry limit by $-\tan ^{2} \theta_{c}[$ [四]:

$$
\begin{aligned}
\frac{A\left(D^{0} \rightarrow \rho^{-} K^{+}\right)}{A\left(D^{0} \rightarrow K^{*-} \pi^{+}\right)} & =\frac{A\left(D^{0} \rightarrow K^{*+} \pi^{-}\right)}{A\left(D^{0} \rightarrow \rho^{+} K^{-}\right)}=\frac{A\left(D^{+} \rightarrow \rho^{+} K^{0}\right)}{A\left(D_{s}^{+} \rightarrow K^{*+} \bar{K}^{0}\right)}=\frac{A\left(D_{s}^{+} \rightarrow K^{*+} K^{0}\right)}{A\left(D^{+} \rightarrow \rho^{+} \bar{K}^{0}\right)} \\
& =\frac{A\left(D^{+} \rightarrow K^{* 0} \pi^{+}\right)}{A\left(D_{s}^{+} \rightarrow \bar{K}^{* 0} K^{+}\right)}=\frac{A\left(D_{s}^{+} \rightarrow K^{* 0} K^{+}\right)}{A\left(D^{+} \rightarrow \bar{K}^{* 0} \pi^{+}\right)}=-\tan ^{2} \theta_{c} .
\end{aligned}
$$

The first two ratios can be tested in an ongoing study by the CLEO Collaboration of the Dalitz plot in $D^{0} \rightarrow K^{+} \pi^{-} \pi^{0}$ [17. The measured value of the penultimate ratio [3], taking into account an $\mathrm{SU}(3)$ breaking phase space factor $p_{\text {c.m. }}^{3} / M_{\text {initial }}^{2}$,

$$
\frac{\left|A\left(D^{+} \rightarrow K^{* 0} \pi^{+}\right)\right|}{\left|A\left(D_{s}^{+} \rightarrow \bar{K}^{* 0} K^{+}\right)\right|}=(1.25 \pm 0.33) \tan ^{2} \theta_{c}
$$

is in agreement with the above prediction.

Predictions very similar to Eq. (8) hold for ratios of the three partial wave amplitudes in decays to two vector mesons. One simply replaces $K \leftrightarrow K^{*}$ and $\pi \leftrightarrow \rho$, 
excluding final states involving $\rho^{0}$ in ratios of $\mathrm{P}$-wave amplitudes. Two triangle relations similar to (9) and (10) are obeyed by $\mathrm{S}$ and $\mathrm{D}$ wave amplitudes, where the two vector mesons are in symmetric U-spin states

$$
\begin{gathered}
A_{S, D}\left(D^{0} \rightarrow K^{*+} \rho^{-}\right)+\sqrt{2} A_{S, D}\left(D^{0} \rightarrow K^{* 0} \rho^{0}\right)=A_{S, D}\left(D_{s}^{+} \rightarrow K^{* 0} K^{*+}\right) \\
A_{S, D}\left(D^{+} \rightarrow K^{* 0} \rho^{+}\right)+\sqrt{2} A_{S, D}\left(D^{+} \rightarrow K^{*+} \rho^{0}\right)=A_{S, D}\left(D_{s}^{+} \rightarrow K^{* 0} K^{*+}\right)
\end{gathered}
$$

Finally, U-spin predictions can be generalized to any U-spin related pair of multibody DCS and CF charmed meson decays. For instance, one predicts for the ratio of nonresonant three-body amplitudes

$$
\frac{A\left(D^{+} \rightarrow K^{+} \pi^{+} \pi^{-}\right)_{\text {nonresonant }}}{A\left(D_{s}^{+} \rightarrow K^{+} K^{-} \pi^{+}\right)_{\text {nonresonant }}}=-\tan ^{2} \theta_{c} .
$$

Taking into account an $\mathrm{SU}(3)$ breaking three-body phase space factor of 1.58 in favor of $D^{+}$decay, the measured value of this ratio [3]

$$
\frac{\left|A\left(D^{+} \rightarrow K^{+} \pi^{+} \pi^{-}\right)_{\text {nonresonant }}\right|}{\left|A\left(D_{s}^{+} \rightarrow K^{+} K^{-} \pi^{+}\right)_{\text {nonresonant }}\right|}=(1.75 \pm 0.59) \tan ^{2} \theta_{c}
$$

is consistent with this prediction although experimental errors are still large. Other predictions [18, such as $A\left(D^{+} \rightarrow K^{+} \pi^{+} \pi^{-}\right) / A\left(D^{+} \rightarrow K^{-} \pi^{+} \pi^{+}\right)=A\left(D_{s}^{+} \rightarrow\right.$ $\left.K^{+} K^{+} \pi^{-}\right) / A\left(D_{s}^{+} \rightarrow K^{+} K^{-} \pi^{+}\right)=-\tan ^{2} \theta_{c}$, where initial and final states in DCS and $\mathrm{CF}$ processes are not related by U-spin, do not follow from $\mathrm{SU}(3)$ alone but require further dynamical assumptions.

Now let us discuss how U-spin breaking may show up in magnitudes of amplitudes and in their final state phase differences. For this matter consider, for instance, the two U-spin related triangles of Eqs. (2) and (9), where the first triangle follows from isospin symmetry while the second one holds only in the $\mathrm{SU}(3)$ symmetry limit. In this limit the two triangles are congruent to each other; the ratio of their corresponding sides is given by a common factor of $-\tan ^{2} \theta_{c}$.

In order to demonstrate the effect of $\mathrm{SU}(3)$ breaking on the pattern of the DCS triangle (9) relative to the $\mathrm{CF}$ triangle (21), it is convenient to decompose amplitudes into diagramatic contributions [19]: a color favored "tree" amplitude $T$, a "color-suppressed" amplitude $\mathrm{C}$ and an "exchange" amplitude $E$. Thus, $\Delta S=\Delta C$ amplitudes can be expressed as

$$
\begin{aligned}
& A\left(D^{0} \rightarrow K^{-} \pi^{+}\right)=T+E, \quad \sqrt{2} A\left(D^{0} \rightarrow \bar{K}^{0} \pi^{0}\right)=C-E \\
& A\left(D^{+} \rightarrow \bar{K}^{0} \pi^{+}\right)=T+C,
\end{aligned}
$$

while $\Delta S=-\Delta C$ amplitudes are

$$
\begin{aligned}
A\left(D^{0} \rightarrow K^{+} \pi^{-}\right) & =-\tan ^{2} \theta_{c}\left(T^{\prime}+E^{\prime}\right), \quad \sqrt{2} A\left(D^{0} \rightarrow K^{0} \pi^{0}\right)=-\tan ^{2} \theta_{c}\left(C^{\prime}-E^{\prime}\right) \\
A\left(D_{s}^{+} \rightarrow K^{0} K^{+}\right) & =-\tan ^{2} \theta_{c}\left(T^{\prime}+C^{\prime}\right),
\end{aligned}
$$

where in the $\mathrm{SU}(3)$ limit $T^{\prime}=T, C^{\prime}=C, E^{\prime}=E$. We stress again that Eqs. (17) and (18) are equivalent to isospin and $\mathrm{SU}(3)$ decompositions, respectively. The above two 
sets of equations provide a suitable phenomenological framework for incorporating important resonant contributions through the terms $E$ and $E^{\prime}$ [7, 20].

A successful fit to all CF $D$ decays to two pseudoscalars, including decays into $K \eta$ and $K \eta^{\prime}$, yields [20]

$$
T=2.69, \quad C=-1.96 e^{i 28^{\circ}}, \quad E=1.60 e^{i 114^{\circ}},
$$

where amplitudes are given in units of $10^{-6} \mathrm{GeV}$. The sizable magnitude of $E$ and its large phase relative to $T$ (chosen to be real) prove the importance of nearby resonances. The color-suppressed amplitude $C$ acquires a smaller complex phase from rescattering through states fed by $T$. We note that also the exotic amplitudes $A\left(D^{+} \rightarrow \bar{K}^{0} \pi^{+}\right)$and $A\left(D_{s}^{+} \rightarrow K^{0} K^{+}\right)$, which do not include resonance contributions, carry final state phases. The lengths of the three sides of the CF triangle (2) from which the amplitudes (19) were obtained [20] are

$$
|T+E|=2.50, \quad|C-E|=2.62, \quad|T+C|=1.36 \quad,
$$

and the corresponding angles opposite these sides are $70^{\circ}, 80^{\circ}$ and $30^{\circ}$, respectively.

$\mathrm{SU}(3)$ breaking is introduced in $T^{\prime}$ and $C^{\prime}$ by assuming factorization and using the above mentioned value $F_{0}^{D K}\left(m_{\pi}^{2}\right) / F_{0}^{D \pi}\left(m_{K}^{2}\right) \approx F_{0}^{D K}(0) / F_{0}^{D \pi}(0)=1.0$ [6], where a few percent correction due to an extrapolation from the measured value at $q^{2}=0$ is neglected. Thus

$$
\frac{T^{\prime}}{T}=\frac{f_{K} F_{0}^{D \pi}\left(m_{K}^{2}\right)\left(1-m_{\pi}^{2} / m_{D}^{2}\right)}{f_{\pi} F_{0}^{D K}\left(m_{\pi}^{2}\right)\left(1-m_{K}^{2} / m_{D}^{2}\right)}=1.31 \quad, \quad \frac{C^{\prime}}{C}=1 .
$$

For the resonant contribution we assume six possible factors which cover a reasonable range of parameters: (a) $E^{\prime}=1.3 E$ (b) $E^{\prime}=0.7 E$ (c) $E^{\prime}=1.3 e^{i 30^{\circ}} E$ (d) $E^{\prime}=$ $0.7 e^{i 30^{\circ}} E$ (e) $E^{\prime}=1.3 e^{-i 30^{\circ}} E$ and (f) $E^{\prime}=0.7 e^{-i 30^{\circ}} E$. A factor between 0.7 and 1.3 seems adequate for $\mathrm{SU}(3)$ breaking in the $D^{0}$ effective couplings to the resonances and to their charge-conjugates. This factor is real for one dominant resonance around 1800-1900 MeV [7], for which the common phase of $E$ and $E^{\prime}$ depends only on the resonance mass and width. The contribution from the more distant resonance at 1430 $\mathrm{MeV}$ was estimated [7] to be at least a factor two smaller. Even in case that the phase of this contribution is $90^{\circ}$ relative to the dominant one, it may change the phase of $E^{\prime}$ relative to $E$ by no more than $30^{\circ}$ which we consider as extreme cases.

The resulting lengths of sides and the angles in the $\Delta S=-\Delta C$ triangle are shown in Table 1. The four values of $\left|T^{\prime}+E^{\prime}\right| /|T+E|$ in cases (a)-(d) are consistent with the average measurement (1) at one standard deviation, whereas the values (e) and (f) corresponding to a negative $\mathrm{SU}(3)$ breaking phase between $E$ and $E^{\prime}$ are excluded by the data at a high level of confidence. In the four cases which are consistent with data the angles opposite the three sides $T^{\prime}+E^{\prime}, C^{\prime}-E^{\prime}$ and $T^{\prime}+C^{\prime}$ are (a) $80^{\circ}, 63^{\circ}, 37^{\circ}$ (b) $96^{\circ}, 46^{\circ}, 38^{\circ}$ (c) $64^{\circ}, 61^{\circ}, 55^{\circ}$ and (d) $91^{\circ}, 41^{\circ}, 48^{\circ}$. Comparing this with the abovementioned angles of the CF triangle, we see that final state phase patterns in DCS and $\mathrm{CF}$ amplitude triangles can be quite different. Within the above range of $\mathrm{SU}(3)$ breaking parameters corresponding angles in the two triangles differ by as much as $39^{\circ}$. 
Table I: Sides and opposite angles of amplitude triangles. See text for units. First line denotes $\Delta S=\Delta C$ triangle (2); other lines denote $\Delta S=-\Delta C$ triangles (9).

\begin{tabular}{|l|lc|lc|lc|}
\hline$E^{\prime} / E$ & \multicolumn{2}{|c|}{$T+E$ or $T^{\prime}+E^{\prime}$} & \multicolumn{2}{|c|}{$C-E$ or $C^{\prime}-E^{\prime}$} & \multicolumn{2}{|c|}{$T+C$ or $T^{\prime}+C^{\prime}$} \\
(case) & Side & Angle & Side & Angle & Side & Angle \\
\hline CF triangle & 2.50 & $70^{\circ}$ & 2.62 & $80^{\circ}$ & 1.36 & $30^{\circ}$ \\
\hline (a) 1.3 & 3.28 & $80^{\circ}$ & 2.96 & $63^{\circ}$ & 2.02 & $37^{\circ}$ \\
(b) 0.7 & 3.23 & $96^{\circ}$ & 2.32 & $46^{\circ}$ & 2.02 & $38^{\circ}$ \\
(c) $1.3 e^{i 30^{\circ}}$ & 2.21 & $64^{\circ}$ & 2.14 & $61^{\circ}$ & 2.02 & $55^{\circ}$ \\
(d) $0.7 e^{i 30^{\circ}}$ & 2.70 & $91^{\circ}$ & 1.78 & $41^{\circ}$ & 2.02 & $48^{\circ}$ \\
(e) $1.3 e^{-i 30^{\circ}}$ & 4.28 & $96^{\circ}$ & 3.57 & $56^{\circ}$ & 2.02 & $28^{\circ}$ \\
(f) $0.7 e^{-i 30^{\circ}}$ & 3.81 & $105^{\circ}$ & 2.75 & $44^{\circ}$ & 2.02 & $31^{\circ}$ \\
\hline
\end{tabular}

An interesting quantity is the final state phase difference $\delta=\operatorname{Arg}\left[\left(T^{\prime}+E^{\prime}\right) /(T+\right.$ $E)$ ] between $A\left(D^{0} \rightarrow K^{+} \pi^{-}\right)$and $A\left(D^{0} \rightarrow K^{-} \pi^{+}\right)$, which vanishes in the U-spin symmetry limit and which plays an important role in studies of $D^{0}-\bar{D}^{0}$ mixing as mentioned in the introduction. This phase is found to be $0,-17,-2$ and -22 degrees in cases (a) (b) (c) and (d), respectively. It can be positive if $\mathrm{SU}(3)$ breaking enhances $E^{\prime}$ more than $T^{\prime}$. Crudely speaking, very different shapes of the CF and DCS triangles would be evidence for a large value of $|\delta|$. The magnitude of this phase grows with an increasing difference between the two angles opposite $C-E$ and $C^{\prime}-E^{\prime}$. In cases (b) and (d), where this angle difference is $34^{\circ}$ and $39^{\circ}, \delta$ is $-17^{\circ}$ and $-22^{\circ}$, respectively, about half of this angle difference. The other $17^{\circ}=\operatorname{Arg}\left[\left(T^{\prime}+C^{\prime}\right) /(T+C)\right]$ are due to a phase difference between the exotic amplitudes $A\left(D_{s}^{+} \rightarrow K^{0} K^{+}\right)$and $A\left(D^{+} \rightarrow \bar{K}^{0} \pi^{+}\right)$. We note that very large values of $\delta$, around $45^{\circ}$ or larger as envisaged in [1], cannot be accommodated in our scheme within a reasonable range of $\mathrm{SU}(3)$ breaking parameters. Such values would require an $\mathrm{SU}(3)$ breaking factor of 2.5 in $E^{\prime} / E$.

Finally, we make a few comments on the difficulty of measuring two of the amplitudes in Eq. (9). There is no way to distinguish a $K^{0}$ from a $\bar{K}^{0}$ when it is detected as a $K_{S}$. In CF decays one simply assumes the flavor of a neutral kaon which is an adequate approximation. This would be clearly wrong for DCS decays. Tagging the flavor of a $K^{0}$ through the charged lepton $\ell^{+}$in semileptonic decays requires looking at decay times less than a few $K_{S}$ lifetimes, before the $K_{S}$ has decayed away. This reduces the rate by a large factor and does not seem feasible for rare DCS decays.

Alternatively, one may measure the triangles (13) describing DCS decays to two vector mesons in $\mathrm{S}$ and $\mathrm{D}$ waves, where $K^{* 0} \rightarrow K^{+} \pi^{-}$identifies the flavor of the neutral $K^{*}$. The structure of these triangles could then be compared with those of (5) for $\mathrm{S}$ and $\mathrm{D}$ waves. This requires a partial wave analysis through angular distributions of decay products. Such analysis was performed in CF decays [21], where evidence was found for negligible P-wave amplitudes.

Note added: After the submission of this Letter a new result for the ratio of branching ratios in Eq. (11) appeared [22] corresponding to a value $(1.56 \pm 0.34) \tan ^{4} \theta_{c}$. 
We thank Yuval Grossman, Harry Lipkin, Harry Nelson and Alex Smith for useful discussions, and Mikhail Voloshin for reminding us of the very early papers in Ref. [四]. J. L. R. wishes to thank the Technion - Israel Institute of Technology for gracious hospitality during part of this work. This work was supported in part by the United States Department of Energy through Grant No. DE FG02 90ER40560 and by the U. S. - Israel Binational Science Foundation through Grant No. 98-00237.

\section{References}

[1] CLEO Collaboration, R. Godang et al., Phys. Rev. Lett. 84 (2000) 5038.

[2] Particle Data Group, C. Caso et al., Eur. Phys. J. C 3 (1998) 1; see also ALEPH Collaboration, R. Barate et al., Phys. Lett. B 436 (1998) 211.

[3] Particle Data Group, D. E. Groom et al., Eur. Phys. J. C 15 (2000) 1.

[4] R. L. Kingsley, S. B. Trieman, F. Wilczek and A. Zee, Phys. Rev. D 11 (1975) 1919; M. B. Voloshin, V. I. Zakharov and L. B. Okun, Pis'ma Zh. Eksp. Teor. Fiz. 21 (1975) 403 [JETP Lett. 21 (1975) 183].

[5] I. Bigi and A. I. Sanda, Phys. Lett. B 171 (1986) 320; L. L. Chau and H. Y. Cheng, Phys. Lett. B 333 (1994) 514; F. Buccella, M. Lusignoli, G. Miele, A. Pugliese and P. Santorelli, Phys. Rev. D 51 (1995) 3478.

[6] Mark III Collaboration, J. Adler et al., Phys. Rev. Lett. 62 (1989) 1821; CLEO Collaboration, F. Butler et al., Phys. Rev. D 52 (1995) 2656; E687 Collaboration, P. L. Frabetti et al., Phys. Lett. B 382 (1996) 312; CLEO Collaboration, J. Bartelt et al., Phys. Lett. B 405 (1997) 373.

[7] M. Gronau, Phys. Rev. Lett. 83 (1999) 4005.

[8] For earlier discussions of resonance effects see, H. J. Lipkin, Phys. Rev. Lett. 44 (1980) 710; C. Sorensen, Phys. Rev. D 23 (1981) 2618; E. Golowich, Phys. Rev. D 24 (1981) 676; A. N. Kamal and E. D. Cooper, Zeit. Phys. C 8 (1981) 67; A. N. Kamal, Int. J. Mod. Phys. A 7 (1992) 3515; F. E. Close and H. J. Lipkin, Phys. Lett. B 372 (1996) 306; E. Golowich and A. A. Petrov, Phys. Lett. B 427 (1998) 172.

[9] Flavor SU(3) breaking in charmed particle decays has been considered by many authors. See, e.g., V. Barger and S. Pakvasa, Phys. Rev. Lett. 43 (1979) 812; A. N. Kamal and R. C. Verma, Phys. Rev. D 35 (1987) 3515; 36 (1987) 3527(E); R. C. Verma and A. N. Kamal, Phys. Rev. D 43 (1991) 829; L. L. Chau and H. Y. Cheng, Phys. Rev. D 36 (1987) 137; Phys. Lett. B 280 (1992) 281; I. Hinchliffe and T. A. Kaeding, Phys. Rev. D 54 (1996) 914.

[10] Chau and Cheng, Ref. [5]; M. Gronau and D. Pirjol, Phys. Rev. D 62 (2000) 077301 . 
[11] S. Bergmann, Y. Grossman, Z. Ligeti, Y. Nir and A. A. Petrov, Phys. Lett. B 486 (2000) 418. Here it was noted that a very large value of $\delta$ may be required to resolve a possible discrepancy between the result of Ref. [1] and that of FOCUS Collaboration, J. M. Link, et al., Phys. Lett. B 485 (2000) 62.

[12] L. Wolfenstein, Phys. Rev. Lett. 75 (1995) 2460.

[13] Chau and Cheng, Ref. [5]; T. E. Browder and S. Pakvasa, Phys. Lett. B 383 (1996) 475; A. F. Falk, Y. Nir and A. A. Petrov, JHEP 9912 (1999) 019.

[14] U-spin predictions in charmless $B$ decays were studied recently by M. Gronau, Phys. Lett. B 492 (2000) 297.

[15] Mark III Collaboration, J. Adler et al., Phys. Lett. B 196 (1987) 107; Fermilab E691 Collaboration, J. C. Anjos et al., Phys. Rev. D 48 (1993) 56; ARGUS Collaboration, H. Albrecht et al., Phys. Lett. B 308 (1993) 435; Fermilab E687 Collaboration, P. Frabetti et al., Phys. Lett. B 331 (1994) 217; CLEO Collaboration, M. Bishai et al., Phys. Rev. Lett. 78 (1997) 3261.

[16] For a recent update, see J. L. Rosner, Phys. Rev. D 60 (1999) 074029; see also M. Suzuki, Phys. Rev. D 58 (1998) 111504.

[17] H. Nelson and A. Smith, private communications.

[18] H. J. Lipkin and Z. Z. Xing, Phys. Lett. B 450 (1999) 405; H. J. Lipkin, Phys. Lett. B 494 (2000) 248.

[19] L. L. Chau and H. Y. Cheng, Phys. Rev. D 36 (1987) 137; M. Gronau, O. F. Hernández, D. London and J. L. Rosner, Phys. Rev. D 50 (1994) 4529.

[20] J. L. Rosner, Phys. Rev. D 60 (1999) 114026.

[21] Mark III Collaboration, D. M. Coffman et al., Phys. Rev. D 45 (1992) 2196; E691 Collaboration, J. C. Anjos et al., Phys. Rev. D 46 (1992) 1941.

[22] FOCUS Collaboration, J. M. Link et al., hep-ex/0012048. 